\title{
TCRP1 induces tamoxifen resistance by promoting the activation of SGK1 in MCF-7 cells
}

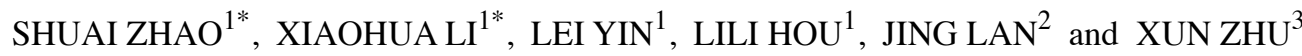 \\ ${ }^{1}$ Department of Breast and Thyroid Surgery, Wuzhong People's Hospital of Suzhou City, \\ Suzhou, Jiangsu 215128; ${ }^{2}$ Department of General Surgery, The First Hospital Affiliated of Suzhou University, \\ Suzhou, Jiangsu 215006; ${ }^{3}$ Department of Breast and Thyroid Surgery, \\ The Second Affiliated Hospital of Suzhou University, Suzhou, Jiangsu 215004, P.R. China
}

Received August 30, 2019; Accepted March 4, 2020

DOI: $10.3892 /$ or.2020.7577

\begin{abstract}
Tamoxifen is widely used as a highly effective drug for treating estrogen-receptor (ER) alpha-positive breast cancer. However, tamoxifen resistance developed during cancer treatment remains a significant challenge. Tongue cancer resistance-related protein1 (TCRP1), which is recognized as a novel drug target, is related to chemo-resistance in human cancers, moreover, it is often overexpressed in various cancer cells, such as in lung cancer, breast cancer, and tongue cancer. However, the effects of TCRP1 on tamoxifen-resistant breast cancer cells and tissues are far from clear. The present study revealed that TCRP1 induced tamoxifen resistance in breast cancer cells. Western blotting, quantitative real-time polymerase chain reaction (RT-PCR) and immunohistochemical staining were performed to detect the expression level of TCRP1 in vivo and in vitro between primary breast cancer tissues and tamoxifen-resistant breast cancer tissues. The data revealed that the expression of
\end{abstract}

Correspondence to: Dr Jing Lan, Department of General Surgery, The First Hospital Affiliated of Suzhou University, 899 Pinghai Road, Suzhou, Jiangsu 215006, P.R. China

E-mail: kakahaha_2008@qq.com

Dr Xun Zhu, Department of Breast and Thyroid Surgery, The Second Affiliated Hospital of Suzhou University, 1055 San Xiang Road, Suzhou, Jiangsu 215004, P.R. China

E-mail: zhuxunsdfey@163.com

*Contributed equally

Abbreviations: TCRP1, tongue cancer resistance-related protein 1; PDK1, phosphoinositide-dependent kinase 1; SGK1, glucocorticoid-inducible kinase 1; DMEM, Dulbecco's modified Eagle's medium; FBS, fetal bovine serum; cDNA, complementary DNA; ER, estrogen receptors; mRNA, messenger RNA; PVDF, polyvinylidene difluoride; shRNA, short hairpin RNA; GAPDH, glyceraldehyde-3-phosphate dehydrogenase; NSh, non-short hairpin

Key words: breast cancer, tamoxifen-resistant, tongue cancer resistance-related protein1, glucocorticoid-inducible kinase 1, MCF-7 cells
TCRP1 was upregulated in the tamoxifen-resistant breast cancer tissues and human breast cancer cell line, MCF-7. Further study revealed that knocking down TCRP1 inhibited the growth of MCF-7 cells with tamoxifen-resistance (MCF7-R cells) and induced cell apoptosis. Moreover, TCRP1 promoted serum- and glucocorticoid-inducible kinase 1 (SGK1) activation via phosphorylation of phosphoinositide-dependent kinase 1 (PDK1) in MCF7-R cells. In addition, it was also observed that knocking down TCRP1 inhibited tumorigenesis of MCF-7 cells in nude mice. In conclusion, these data indicated that TCRP1 could induce tamoxifen resistance by regulating the PDK1/SGK1 signaling pathway. Thus, TCRP1 could be explored as a promising candidate for treating tamoxifen-resistant breast cancer in the future.

\section{Introduction}

Breast cancer is a malignant tumor with the highest incidence and mortality for women in the world. Annually, approximately 1 million new cases of breast cancer are diagnosed worldwide (1). According to the global cancer statistics of 2018 , the mortality of breast cancer accounted for $11.6 \%$ of all cancer-related mortalities (2).

Estrogen signaling pathway functions critically in the pathogenesis of breast cancer, and $75 \%$ of breast cancer patients are estrogen receptors (ER) alpha-positive and are generally treated by endocrine therapy $(3,4)$. Tamoxifen, which is used as a first-line drug for endocrine therapy, has been extensively used to treat early and late $\mathrm{ER}^{+}$breast cancer patients for the past three decades (5). The structure of tamoxifen is similar to that of estrogen, a selective estrogen receptor regulator (6-10). Although numerous breast cancer patients benefit from the drug, tamoxifen resistance developed during the therapy still remains a clinical challenge (11). Approximately $1 / 3$ of patients develop resistance to tamoxifen at the beginning of treatment, and those who initially are sensitive to the drug will later develop resistance (12). Thus, investigating the mechanisms underlying the resistance and detecting the molecular mediators of tamoxifen are necessary, since it may help identify new strategies against tamoxifen-resistant cancer cells.

TCRP1, alternatively known as FAM168A, is located on human chromosome 11q13.4. It encodes 235 amino acids 
of putative protein and its molecular weight is $25 \mathrm{kDa}$ (13). TCRP1 is used as a novel candidate inducing drug resistance, and it is widely expressed in numerous types of cancer cells (14-16). In the past few years, certain studies have reported that TCRP1 promotes tumorigenesis in oral squamous cell carcinoma through the Akt signaling pathway $(14,15)$. Several studies have reported that TCRP1 mediates DDP-resistant lung cancer cells by inhibiting the degradation of Pol b (16). Moreover, TCRP1 plays an indispensable role in promoting the transformation of NIH/3T3 cells by overexpressing PDK1 and AKT1 (17). However, the effects of TCRP1 on MCF7-R cells and the underlying mechanism are still unknown. The present research, aimed to explore whether TCRP1 mediated tamoxifen resistance in breast cancer and investigated the mechanism involved in the TCRP1-mediated tamoxifen resistance in breast cancer.

\section{Materials and methods}

Cell culture. The human breast adenocarcinoma cell line MCF-7 was purchased from the American Type Culture Collection. The MCF-7 cells were cultured in Dulbecco's modified Eagle medium (DMEM) (Invitrogen; Thermo Fisher Scientific, Inc.) containing 10\% fetal bovine serum (FBS) (Sigma-Aldrich; Merck KGaA) and 1\% penicillin/strptomycin with $5 \% \mathrm{CO}_{2}$ at $37^{\circ} \mathrm{C}$. For the acquired tamoxifen-resistant breast cancer cells, MCF-7 cells were cultured in DMEM containing 10\% FBS with $1 \mu \mathrm{M}$ tamoxifen (cat. no. T5648; Sigma-Aldrich; Merck KGaA) for 3 months, and then an extra $3 \mu \mathrm{M}$ tamoxifen was added into the medium, and incubated for more than 9 months (18). MCF-7 cells and those with tamoxifen resistance (MCF7-R cells) served as tumor cell models.

Quantitative real-time polymerase chain reaction (RT-PCR). Total RNAs were extracted from MCF-7 cells and MCF7-R cells respectively using RNAiso plus reagent (code. no. 9109; Takara), and complementary DNAs (cDNAs) were reverse-transcribed using the PrimeScript ${ }^{\mathrm{TM}} \mathrm{RT}$ Master Mix (Perfect Real Time) (code no. RR036) in a 10- $\mu$ l reaction system, which consisted of $500 \mathrm{ng}$ total RNAs, $2 \mu \mathrm{l} 5 \mathrm{X}$ PrimeScript RT Master Mix (Perfect Real-Time), and $10 \mu \mathrm{l}$ RNase-free $\mathrm{ddH}_{2} \mathrm{O}$. The reaction was performed at $50^{\circ} \mathrm{C}$ for $15 \mathrm{~min}$ and at $85^{\circ} \mathrm{C}$ for $2 \mathrm{~min}$. The cDNAs were diluted to a ratio of 1:4 by $\mathrm{ddH}_{2} \mathrm{O}$ before using. According to a q-PCR assay, $10 \mu 1$ reaction systems (cat. no. 04913914001; Roche Applied Science), which were composed of $5 \mu \mathrm{l}$ FastStart Universal SYBR Green Master, $2 \mu 1 \mathrm{cDNA}, 2 \mu 1 \mathrm{ddH}_{2} \mathrm{O}$, and $1 \mu \mathrm{l}$ primer, were performed. The results were analyzed by Rotor-Gene 5 software (Rotor-Gene; Corbett Research). The reaction was carried out at $50^{\circ} \mathrm{C}$ for $2 \mathrm{~min}$ and at $95^{\circ} \mathrm{C}$ for $10 \mathrm{~min}$, then at $95^{\circ} \mathrm{C}$ for $20 \mathrm{sec}, 65^{\circ} \mathrm{C}$ for $20 \mathrm{sec}$ and $72^{\circ} \mathrm{C}$ for $30 \mathrm{sec}$ for amplification, for a total of 40 cycles. Glyceraldehyde-3-phosphate dehydrogenase (GAPDH) RNA served as an internal reference. Primers used for quantitative real-time PCR were as follows: TCRP1 forward, 5'-CTCAGC CTCTTGCTGTGATG-3' and reverse, 5'-AACTGTGCCCAT CCTACCAG-3'; GAPDH forward, 5'-TGCACCACCAAC TGCTTAGC-3' and reverse, 5'-GGCATGGACTGTGGTCAT GA-3'; SGK1 forward, 5'-GCAGAAGAAGTGTTCTATGCA GT-3' and reverse, 5'-CCGCTCCGACATAATATGCTT-3'.
Western blotting. Proteins were lysed from a buffer containing Pierce Protease and Phosphatase Inhibitor Mini Tablets. The whole cell lysates were gently transferred from the plate into a $1.5-\mathrm{ml} \mathrm{EP}$ tube, lysed at $4^{\circ} \mathrm{C}$ for $30 \mathrm{~min}$, and then centrifuged at $12,000 \times \mathrm{g}$ for $15 \mathrm{~min}$ at $4^{\circ} \mathrm{C}$. The supernatant was collected from the newly labeled EP tube, and a small amount of the supernatant was used to assess the protein concentration using a BCA Protein Assay Kit (cat. no. 23235; Thermo Fisher Scientific, Inc.). The remaining supernatant was added to the $5 \mathrm{X}$ protein loading buffer at 1:4, and the proteins were boiled at $100^{\circ} \mathrm{C}$ for $5 \mathrm{~min}$. Next, the proteins $(30 \mu \mathrm{g})$ were electrophoretically separated on 10\% SDS-PAGE, and transferred to polyvinylidene difluoride (PVDF) (cat. no. OPVH00010; EMD Millipore) membranes, which were then blocked by $5 \%$ non-fat milk for $1 \mathrm{~h}$ and incubated with a primary antibody at a dilution of 1:1,000 in $1 \mathrm{X}$ TBS buffer at $4^{\circ} \mathrm{C}$ overnight. After washing the membranes with $1 \mathrm{X}$ TBST three times, a secondary antibody conjugated with goat anti-rabbit (cat. no. A0208; Beyotime Institute of Biotechnology) or goat anti-mouse HRP at a dilution of 1:1,000 (cat. no. A0216; Beyotime Institute of Biotechnology) was incubated with the membranes at room temperature for $2 \mathrm{~h}$. The membranes were washed by $1 \mathrm{X}$ TBST again, and protein signals were detected by ECL kit (cat. no. 34577; Thermo Fisher Scientific, Inc.) with a BioRad GS-800 ${ }^{\mathrm{TM}}$ densitometer scanner (Bio-Rad Laboratories), and the data was analyzed by using PDQuest 7.2.0 software (Bio-Rad Laboratories). The antibodies used were as follows: TCRP1 (cat. no. HPA037580; rabbit; 1:200; Sigma-Aldrich; Merck KGaA), PCNA (cat. no. 13110; rabbit; 1:1,000), cleaved caspase-3 (cat. no. 9664; rabbit; 1:1,000), cleaved PARP (cat. no. 5625; rabbit; 1:1,000), p-PDK1 (cat. no. 3438; rabbit, 1:1,000), PDK1 (cat. no. 3062; rabbit, 1:1,000; all from Cell Signaling Technology, Inc.), p-SGK1 (cat. no. SAB4503834; rabbit; 1:1,000; Sigma-Aldrich; Merck KGaA), SGK1 (cat. no. S5188; rabbit; 1:2,000; Sigma-Aldrich; Merck KGaA), and $\beta$-actin (cat. no. 3700, mouse; 1:1,000; Cell Signaling Technology, Inc.).

Cytotoxicity assay. A single cell suspension was prepared from culture medium containing $10 \%$ FBS, and seeded into a 96-well plate at a volume of 5,000 cells/well. After culturing for 3 to 5 days under the same general culture conditions, $20 \mu 1$ MTT solution (cat. no. ST316; Beyotime Institute of Biotechnology) (5 mg/ml with PBS) was added into each well. After incubating the plate at $37^{\circ} \mathrm{C}$ for $2 \mathrm{~h}$, the culture was terminated, and the culture supernatant in the well was carefully discarded. DMSO $(150 \mu \mathrm{l})$ was added into each well and shaken gently for $10 \mathrm{~min}$ to fully melt the crystals. The light absorption value of each well was measured at an absorbance of $490 \mathrm{~nm}$ using a microplate reader on an enzyme-linked immunosorbent monitor. Then the result was recorded, and a cell growth curve was plotted, with time as the abscissa and absorbance as the vertical coordinate.

Short hairpin RNA (shRNA) and plasmid and cell transfection. TCRP1 shRNA (forward primer, GGAAAUACAUAGACC UACA and reverse primer, UGUAGGUCUAUGUAUUUCC) oligonucleo-tides with 3'dTdT overhangs were synthesized by QIAGEN. Control shRNA in the experiments refers to a non-short hairpin (NSh) shRNA (NSF, UUCUCCGAACGUG 
UCACGU; NSR, ACGUGACACGUUCGGAGAA), which was designed and synthesized by QIAGEN. The TCRP1 overexpression vector pcDNA3.1-TCRP1 was constructed as previously described (13). The vector for overexpressing PDK1 (pcDNA3.1-PDK1) and SGK1 (pcDNA3.1-SGK1) were purchased from Shanghai GenePharma Co., Ltd. MCF7-R cells were transfected with shRNA or plasmids by using Lipofectamine 2000 reagent (cat. no. 11668019; Invitrogen; Thermo Fisher Scientific, Inc.). The expression levels of TCRP1, PDK1 and SGK1 were determined by western blotting and q-PCR.

Immunohistochemical staining. Fresh specimens of the primary breast cancer tissues and tamoxifen-resistant breast cancer tissues were collected from Wuzhong People's Hospital. All patients signed a written informed consent, and the present study was approved by the Ethics Committee of Wuzhong People's Hospital, Suzhou, China. The clinical data of patients are presented in Table I. A total of 40 samples were collected and divided into two groups, with 20 samples in each group. The tumor specimens were fixed by $4 \%$ neutral formalin, embedded in paraffin and sectioned into $4-\mu \mathrm{m}$ thick sections by standard SP method. Immunostaining was performed with the avidin-biotin-peroxidase complex method (Ultrasensitive ${ }^{\mathrm{TM}}$; MaiXin). Then tissue sections were incubated with TCRP1 antibody (cat. no. HPA037580; rabbit; 1:200 Sigma-Aldrich; Merck KGaA), SGK1 antibody (cat. no. S5188; rabbit, 1:2,000; Sigma-Aldrich; Merck KGaA), Ki67 antibody (cat. no. 9129; rabbit; 1:400; Cell Signaling Technology, Inc.) overnight at $4^{\circ} \mathrm{C}$, followed by incubation with a goat anti-rabbit biotinylated secondary antibody (cat. no. SA1020; dilution 1:1,000; Boster Bio) at $4^{\circ} \mathrm{C}$ overnight. The images were analyzed with IPLab 4.0 imaging software (Scanalytics, Inc.).

Clonogenic survival assay. MCF7-R cells were seeded at $1 \times 10^{3}$ cells/well into 6 -well plates and cultured overnight. MCF7-R cells were treated by TCRP1 shRNA or shRNA control for $48 \mathrm{~h}$. After growing for 10 days at $37^{\circ} \mathrm{C}$, the colonies were formed, fixed with $4 \%$ paraformaldehyde, stained with $0.1 \%$ crystal violet (Sigma-Aldrich; Merck KGaA) for $15 \mathrm{~min}$. Colonies containing 50 or more cells were counted under a fluorescence microscope (Leica Microsystems).

Flow cytometry. MCF7-R cells were seeded in a 100-mm plate at $1 \times 10^{6}$ cells and cultured overnight. Then, the cells were treated by TCRP1 shRNA, SGK1 overexpression and shRNA control for $48 \mathrm{~h}$. An Annexin V-FITC Apoptosis Detection Kit (cat. no. AVK250; Strong Biotech Corporation) was used to detect the results. After incubation, the medium was removed, and the cells were washed with cold phosphate-buffered saline and then collected. The supernatant was removed by centrifugation at $300 \mathrm{xg}$ for $5 \mathrm{~min}$ and then the cells were resuspended in $1 \mathrm{X}$ Annexin-binding buffer. Then $5 \mu \mathrm{l}$ of Annexin $\mathrm{V}$ and $1 \mu 1100 \mu \mathrm{g} / \mathrm{ml}$ propidium iodide working solution were added to $100 \mu 1$ of cell suspension and incubated with the cells at room temperature for $15 \mathrm{~min}$. Next, $400 \mu \mathrm{l}$ 1X Annexin-binding buffer was added, mixed gently and kept on ice. A flow cytometer (BD FACSCanto; BD Biosciences) was used to analyze the results. Apoptosis at different stages was explored by gating the respective population on the dot plots. There are four stages of cells, to be more specific, Q1 are necrosis cells Annexin $\mathrm{V}^{-} / \mathrm{PI}^{+}$; $\mathrm{Q} 2$ are late apoptotic cells Annexin $\mathrm{V}^{+} / \mathrm{PI}^{+}$; $\mathrm{Q} 3$ are normal cells Annexin $\mathrm{V}^{-} / \mathrm{PI}$; and $\mathrm{Q} 4$ are early apoptosis cells Annexin $\mathrm{V}^{+} / \mathrm{PI}^{-}$.

Animal studies. All animal studies were approved by the Animal Experimentation Ethics Committee of Wuzhong People's Hospital, Suzhou, China. The animal studies were performed according to corresponding protocols. BALB/c nude mice ( $\mathrm{n}=30$; weight, 18-20 g) (Taconic) aged between 4 to 6 weeks old, were used to construct a subcutaneous xenograft model. In brief, $1 \times 10^{5} \mathrm{MCF}-7$-vector cells and $1 \times 10^{5}$ MCF-7-shTCRP1 cells were subcutaneously inoculated on both sides of the rear back in mice ( $\mathrm{N}=15$ per group) (17). The tumor volume was measured and was indicative of tumor growth.

Statistical analysis. The data are presented as the mean \pm SEM of at least three independent experiments. The statistical differences in xenograft tumor growth in response to MCF-7-shTCRP1 treatment were analyzed by one- or two-way ANOVA, followed by Bonferroni's test. Other P-values were subjected to two-tailed unpaired Student's t-test. P-values $<0.05$ were considered to indicate a statistically significant difference. The data were analyzed by Graphpad Prism5 (GraphPad Software, Inc.). Correlations among the expression levels of TCRP1 and PDK1, SGK1 were determined by Pearson's correlation. $\chi^{2}$ test and Student's t-test were used to compare the distribution of categorical and continuous variables, respectively.

\section{Results}

TCRPI expression is increased in tamoxifen-resistant breast cancer tissues. To explore the potential function of TCRP1 in tamoxifen resistance, the expression of TCRP1 was detected in breast cancer tissues. RT-PCR results revealed that TCRP1 mRNA levels were particularly increased in the tamoxifen-resistant breast cancer samples compared with the primary breast cancer samples (Fig. 1A). Consistently, immunohistochemical staining data revealed that TCRP1 was significantly overexpressed in tamoxifen-resistant tissue samples compared with primary tissue samples (Fig. 1B and C).

TCRP1 induces tamoxifen resistance in MCF7-R cells. Next, it was investigated whether TCRP1 was also increased in MCF-7 cells and MCF7-R cells. Cell viabilities of the two cells exposed to tamoxifen $(0-2 \mu \mathrm{M})$ for $48 \mathrm{~h}$ were detected by MTT assay. The dose-response curves were plotted, and the $\mathrm{IC}_{50}$ values of tamoxifen were determined. The present results revealed that the viability of MCF7-R cells was significantly less affected than that of MCF-7 cells under tamoxifen stimulation $\left(\mathrm{IC}_{50}, 17.44 \mu \mathrm{M}\right)$ (Fig. 2A). Next, the mRNA and protein levels of TCRP1 in MCF-7 cells and MCF7-R cells were detected by RT-PCR and western blotting, respectively. The present data revealed that the expression levels of TCRP1 at the mRNA and protein levels were increased in MCF7-R cells compared with MCF-7 cells (Fig. 2B-D). To further investigate whether TCRP1 was closely related to tamoxifen resistance, the effects of knockdown of TCRP1 and overexpression of 
Table I. Clinical features of primary breast cancer group and tamoxifen-resistance breast cancer group samples.

\begin{tabular}{lccc}
\hline Characteristics & All patients & Primary breast cancer & Tamoxifen-resistant breast cancer \\
\hline Depth of invasion & & & 6 \\
T1+T2 & 15 & 13 & 12 \\
T3+T4 & 25 & & 10 \\
Lymph node metastasis & & 3 & 13 \\
N0 & 13 & 14 & 16 \\
N1+N2+N3 & 27 & & 7 \\
Distant metastasis & & 13 & \\
M0 & 29 & 4 & 6 \\
M1 & 11 & 11 & \\
TNM stage & & 12 &
\end{tabular}

Results were obtained using $\chi^{2}$ test.

A

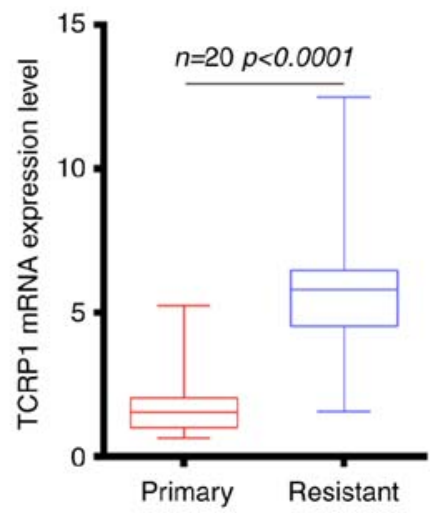

B

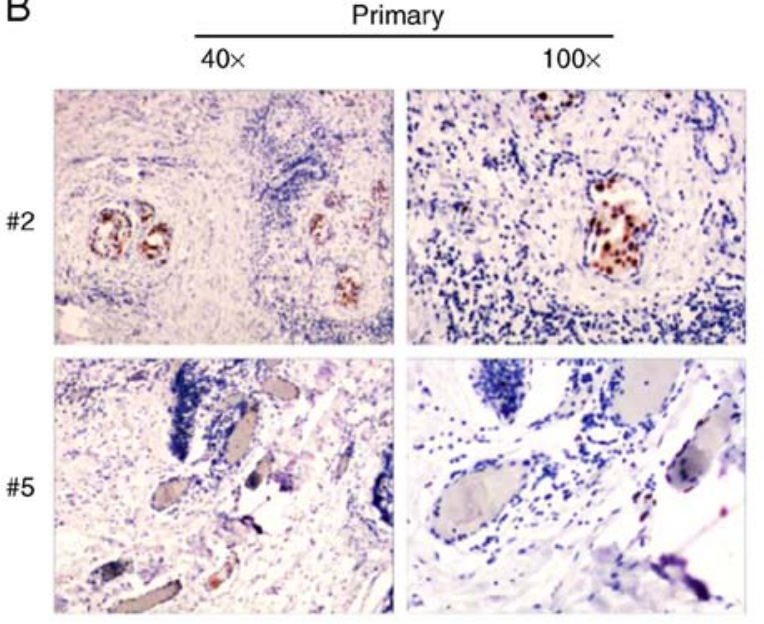

C
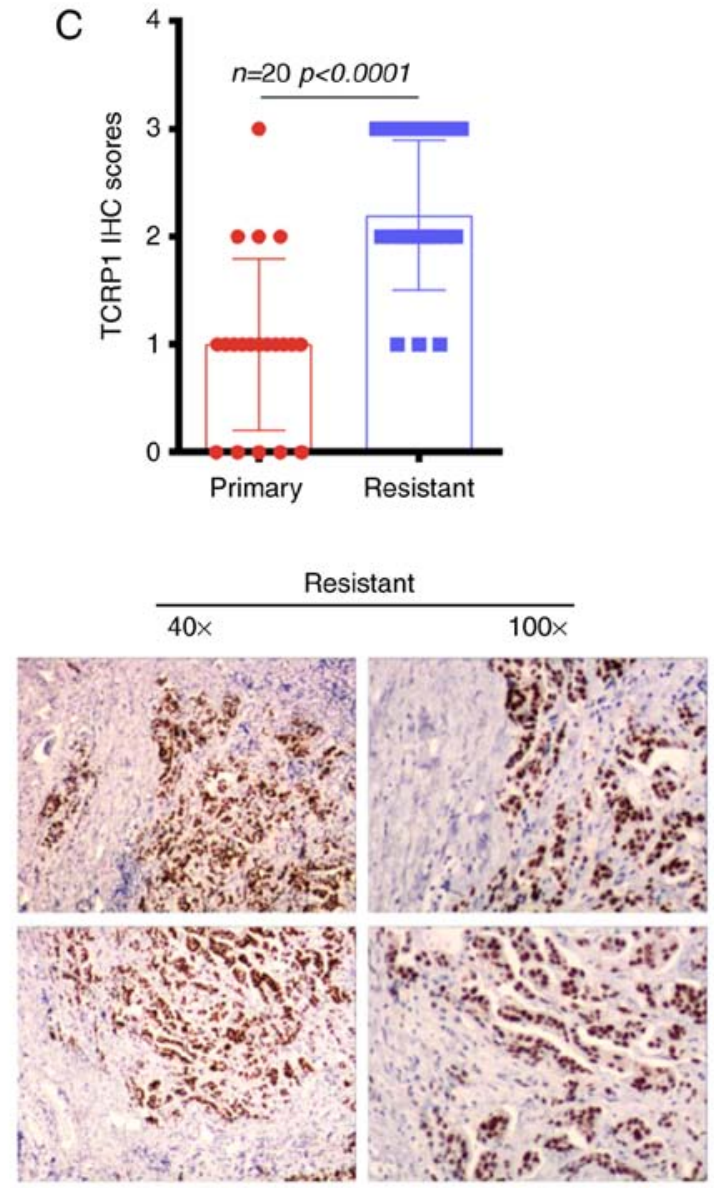

Figure 1. TCRP1 is overexpressed in tamoxifen-resistant breast cancer tissues compared with primary breast cancer tissues. (A) Total mRNAs were extracted from tamoxifen-resistant breast cancer tissues $(\mathrm{n}=20)$ and primary breast cancer tissues $(\mathrm{n}=20)$, respectively. TCRP1 expression was analyzed by RT-PCR. (B and C) Pathological sections in tumor tissues were detected by immunohistochemistry with TCRP1 antibody in the tamoxifen-resistant breast cancer tissues and the primary breast cancer tissues. TCRP1, tongue cancer resistance-related protein1.

TCRP1 were detected on MCF7-R cells. The expression of TCRP1 was determined by RT-PCR and western blotting. The results revealed the short hairpin TCRP1 (shTCRP1) and overexpression of TCRP1 were successfully realized at mRNA and protein levels (Fig. 2E-F), with shTCRP1\#1 exhibiting the strongest knockdown effect, therefore shTCRP1\#1 was used 

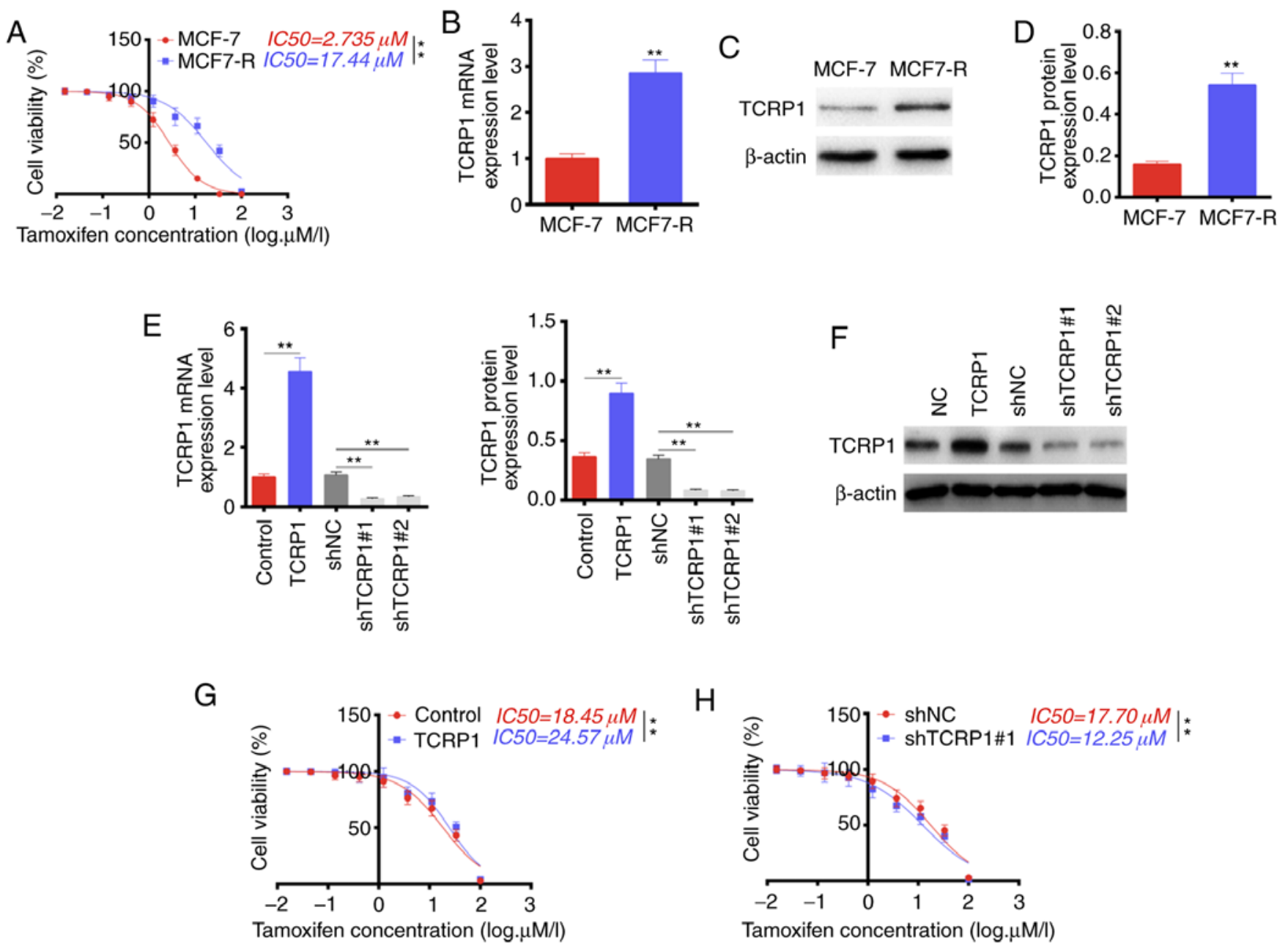

Figure 2. TCRP1 induces tamoxifen resistance in MCF7-R cells. (A) MCF-7 and MCF7-R cells were treated by 0 to $2 \mu \mathrm{M}$ tamoxifen and cultured for $48 \mathrm{~h}$. An MTT assay was performed, and dose-response curves were presented. (B-D) The mRNA and protein expression levels of TCRP1 in MCF-7 and MCF7-R cells were detected by RT-PCR and western blotting. $\beta$-Actin served as an internal control. (E and F) MCF7-R cells were transfected with pcDNA3.1-TCRP1 or control plasmid and treated with 0 to $2 \mu \mathrm{M}$ of tamoxifen for $48 \mathrm{~h}$. MCF7-R cells were transfected with shRNA for TCRP1 or scramble control for $48 \mathrm{~h}$ and then treated with 0 to $2 \mu \mathrm{M}$ tamoxifen for $48 \mathrm{~h}$. The effectiveness of TCRP1 and TCRP1 overexpression were detected by RT-PCR and western blotting. $(\mathrm{G}$ and $\mathrm{H})$ Cell viability was assessed by MTT assay. ${ }^{* *} \mathrm{P}<0.01$. TCRP1, tongue cancer resistance-related protein1.

in the following experiments. The transfected MCF7-R cells were treated with 0 to $2 \mu \mathrm{M}$ tamoxifen, and the result revealed that the $\mathrm{IC}_{50}$ value of TCRP1-overexpressed MCF7-R cells to tamoxifen was higher than the control cells (Fig. 2G). However, the opposite results were revealed in TCRP1-knockdown MCF7-R cells. The $\mathrm{IC}_{50}$ value of TCRP1-knockdown cells to tamoxifen was lower than that of shRNA control cells (Fig. 2H). These data indicated that overexpression of TCRP1 increased resistance to tamoxifen in MCF7-R cells, while knocking down TCRP1 significantly attenuated the tamoxifen resistance in MCF7-R cells.

Knockdown of TCRP1 decreases cell proliferation and promotes apoptosis in MCF7-R cells. It was revealed that TCRP1 induced the tamoxifen resistance in MCF7-R cells, however, the underlying mechanisms were still unclear. To explore the functions of TCRP1 in MCF7-R cells, MCF7-R cells were treated with shRNA for TCRP1 or shRNA control. An MTT assay and clonogenic survival assay were performed to evaluate the effects of TCRP1 on the proliferation of MCF7-R cells. The results revealed that knockdown of TCRP1 significantly decreased cell proliferation (Fig. 3A). Clonogenic survival assay analysis also revealed that clonogenic ability of TCRP1-knockdown cells was significantly attenuated compared with shRNA control cells (Fig. 3B and C). Flow cytometry was performed to examine whether TCRP1 was involved in cell apoptosis, and the results revealed that the proportion of apoptotic cells in the TCRP1-knockdown cells were significantly increased compared with the control cells (Fig. 3D and E). The expression levels of several key apoptosis-associated proteins were further detected, and the data revealed that the pro-apoptotic proteins, cleaved caspase- 3 and cleaved PARP were significantly upregulated, and the level of the anti-apoptotic protein PCNA was downregulated in the TCRP1-knockdown cells (Fig. 3F and G). These findings indicated that knockdown of TCRP1 inhibited the growth of MCF7-R cells, and promoted cell apoptosis.

TCRP1 phosphorylates PDK1 and further activates PDK1/SGK1 signaling. It has been reported that TCRP1 can interact with PDK1, which promotes tumor growth and metastasis in a spontaneous breast cancer model (19). PDK1 activity 
A

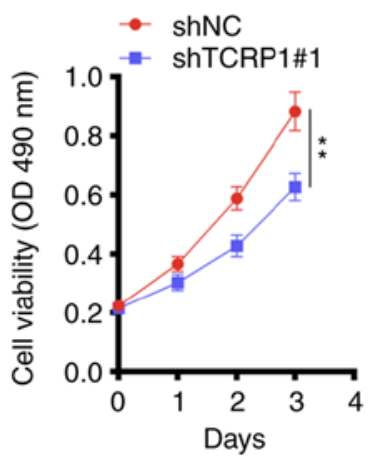

B

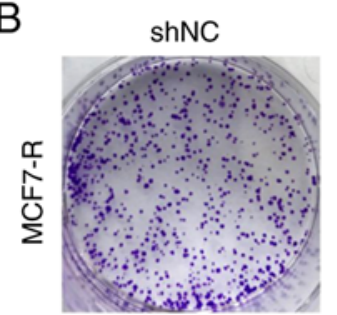

ShTCRP1\#1

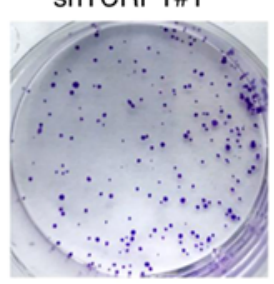

C

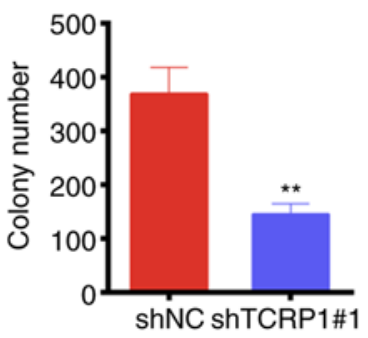

D

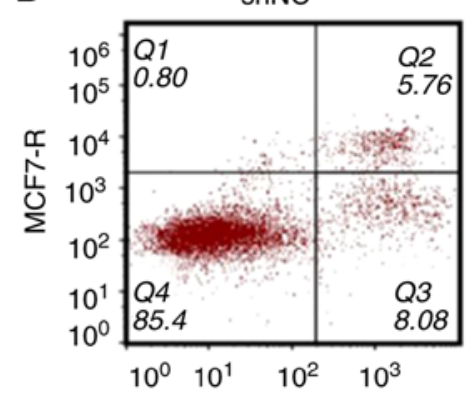

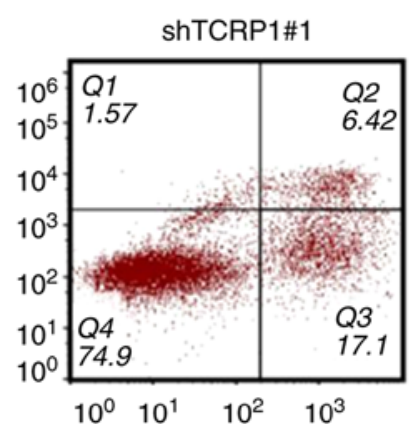

E

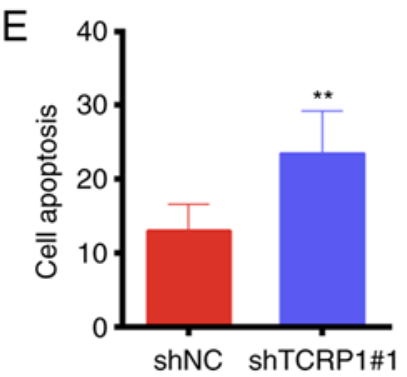

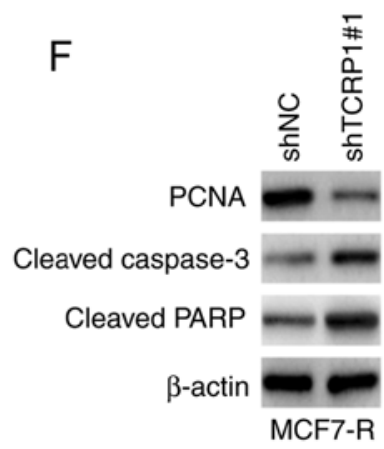

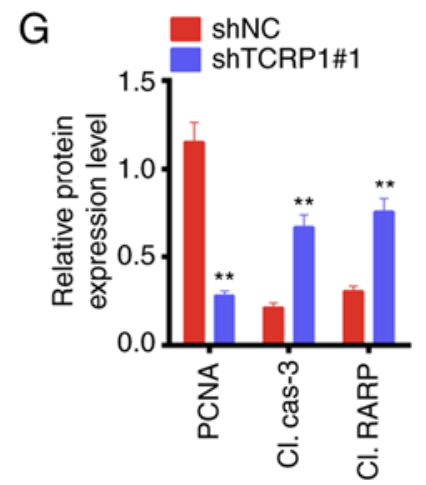

Figure 3. Knockdown of TCRP1 decreases cell proliferation and promotes cell apoptosis in MCF7-R cells. (A) MCF7-R cells were transfected with shRNA for TCRP1 or scramble control and incubated for 0-3 days. (A) Cell viability was detected by MTT assay. (B and C) Clonogenic capacity was assessed by plate cloning assay. (D and E) Cell apoptosis was detected by flow cytometry with Annexin V-FITC/PI dual staining. (F and G) Apoptotic-related proteins were detected by western blotting. ${ }^{* *} \mathrm{P}<0.01$. TCRP1, tongue cancer resistance-related protein1.

was suppressed to inhibit downstream SGK1 phosphorylation and activity in BYL719-resistant cells (20). Therefore, it was explored whether TCRP1 induced tamoxifen resistance in the MCF7-R cells via PDK1/SGK1 signaling. MCF7-R cells were treated with shRNA for TCRP1 or shRNA control, respectively, while MCF7-R cells were transfected with pcDNA3.1-TCRP1 or control plasmid, respectively. The results revealed that phosphorylation levels of PDK1 and SGK1 were significantly increased in TCRP1-overexpressed cells, however, the expression levels of phosphorylated (p)-PDK1 and p-SGK1 were decreased in TCRP1-knockdown cells, compared with shRNA control cells (Fig. 4A and D). In order to further confirm the relationship between PDK1 and SGK1, MCF7-R cells were transfected with PDK1 expression vector or control vector. The results revealed that the p-PDK1 and p-SGK1 were significantly upregulated in MCF7-R cells (Fig. 4B and E). To elucidate the signaling pathway, a co-transfection system was constructed with PDK1 expression vector in combination with the TCRP1 shRNA into MCF7-R cells, and as revealed in Fig. 4C and F, phosphorylation of SGK1 was decreased in TCRP1-knockdown cells compared with shRNA control cells. However, after transfection of PDK1 expression vector into TCRP1-knockdown cells, the protein level of p-SGK1 was restored to the level similar to that of endogenous expression. These data indicated that TCRP1 could activate PDK1/SGK1 signaling by phosphorylating PDK1 and then further phosphorylating SGK1.

TCRP1 induces tamoxifen resistance by activating SGK1. It was further investigated whether SGK1 played a critical role in TCRP1-mediated tamoxifen resistance. MCF7-R cells were treated with SGK1 expression vector, or TCRP1 shRNA, or co-transfection, or control, respectively. The transfection efficiency was detected by q-PCR, and the 

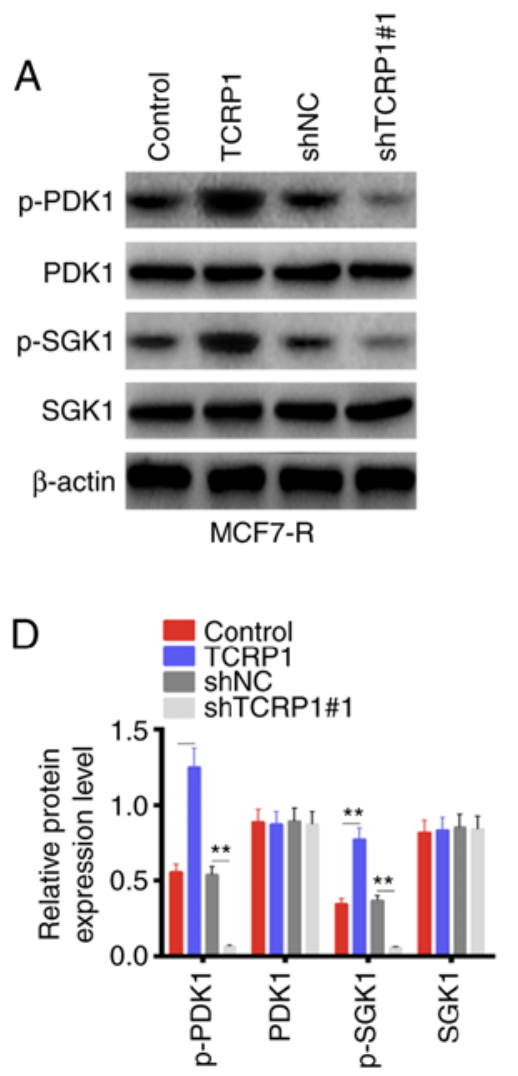

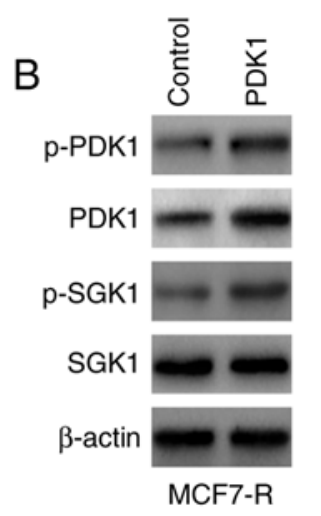

E

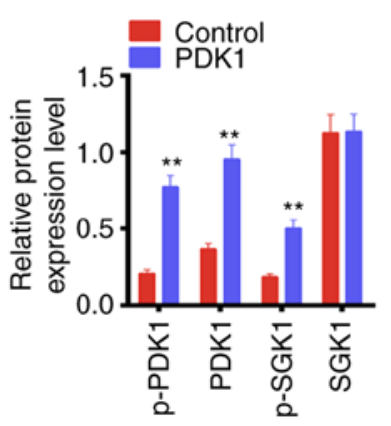

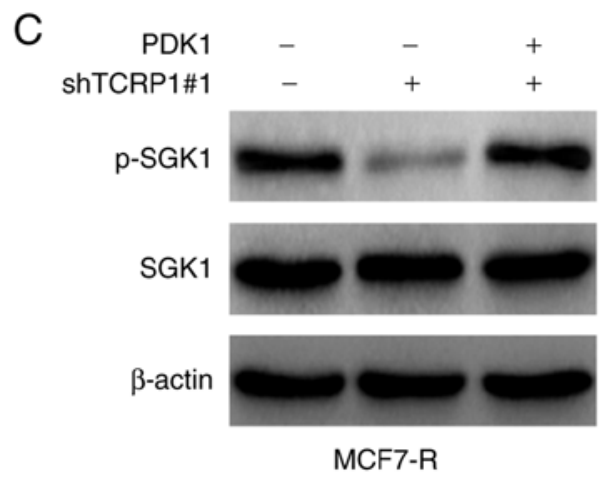

$\mathrm{F}$

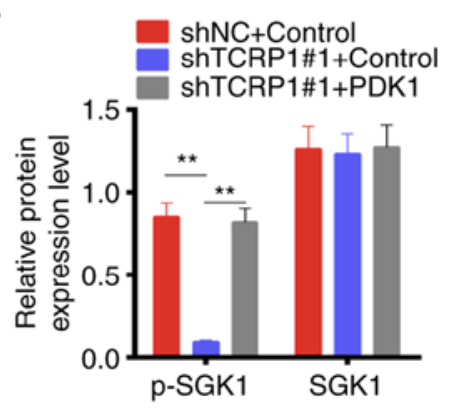

Figure 4. TCRP1 phosphorylates PDK1 and further activates PDK1/SGK1 signaling. (A and D) MCF7-R cells were transfected with pcDNA3.1-TCRP1 or control plasmid. MCF7-R cells were transfected with shRNA for TCRP1 or scramble control for 48 h. The levels of p-PDK1 and PDK1 and p-SGK1 and SGK1 were detected by western blotting. (B and E) MCF7-R cells were transfected with PDK1 or control plasmid for 48 h. The levels of p-PDK1 and PDK1 and p-SGK1 and SGK1 were detected by western blotting. (C and F) MCF7-R cells were transfected with a co-transfection system with PDK1 expression vector in combination with the TCRP1 shRNA into MCF7-R cells or transfected with shRNA for TCRP1 alone or control. The expression of p-SGK1 and SGK1 was detected by western blotting. ${ }^{* *} \mathrm{P}<0.01$. TCRP1, tongue cancer resistance-related protein1; PDK1, phosphoinositide-dependent kinase 1; SGK1, glucocorticoid-inducible kinase 1.

efficiency of SGK1 was revealed to be significantly increased in the SGK1-overexpressed cells compared to the control cells (Fig. 5A). In TCRP1-knockdown cells, the phosphorylation of SGK1 was significantly decreased compared with the control cells. In addition, in TCRP1-knockdown cells treated by SGK1 expression vector, the expression of p-SGK1 was increased to the level similar to that of the endogenous expression (Fig. 5B and C). Moreover, cell viability was detected by MTT assays in the three groups under stimulation of 0 to $2 \mu \mathrm{M}$ tamoxifen. The $\mathrm{IC}_{50}$ value of the TCRP1-knockdown cells was decreased compared to the control cells, however, the $\mathrm{IC}_{50}$ value of the co-transfected cells was increased compared with TCRP1-knockdown cells (Fig. 5D). Furthermore, flow cytometric results revealed that treatment with TCRP1 shRNA promoted cell apoptosis, however, when it was used in combination with overexpressed SGK1, apoptosis was decreased to the control level (Fig. 5E and F). These experiments demonstrated that TCRP1 induced tamoxifen resistance possibly by activating SGK1.

Knockdown of TCRPI inhibits the growth of transplanted tumor in mice. TCRP1-knockdown MCF-7 cells and shRNA control MCF-7 cells were subcutaneously inoculated on both sides of the rear back in nude mice. After feeding the mice for 3 weeks, the treated mice were able to form xenografts. As revealed in Fig. 6A, tumor growth was significantly decreased in the mice treated by the TCRP1 shRNA group compared with that of the shRNA control group. Consistently, the size of control tumors was significantly increased compared to that of TCRP1-knockdown tumors (Fig. 6B). The results of the tumor weight revealed that the xenografts in the TCRP1-knockdown group had a significantly decreased grow th rate compared with that of the control group (Fig. 6C). To further analyze related proliferation indexes of tumor growth, immunohistochemistry with Ki67 staining was performed. The results revealed that the amount of Ki67-positive staining in the TCRP1-knockdown group was significantly decreased compared to that of the control group (Fig. 6D). Moreover, the expression of TCRP1 and SGK1 was increased (Fig. 6D). Thus, these results indicated that TCRP1 inhibited the proliferation of MCF7-R cells in vitro and in vivo.

\section{Discussion}

The present study aimed to explore the function and mechanism involved in TCRP1-mediated tamoxifen resistance in MCF7-R cells. The present results revealed that TCRP1 plays a vital role in tamoxifen resistance in MCF7-R cells, and that knockdown of TCRP1 decreased tamoxifen resistance, inhibited the growth of MCF7-R cells, and promoted cell apoptosis. 

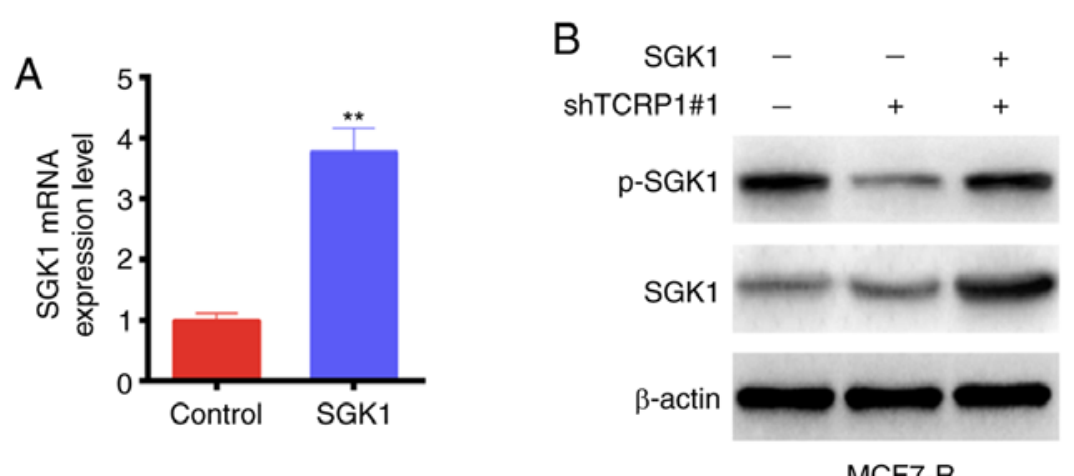

MCF7-R
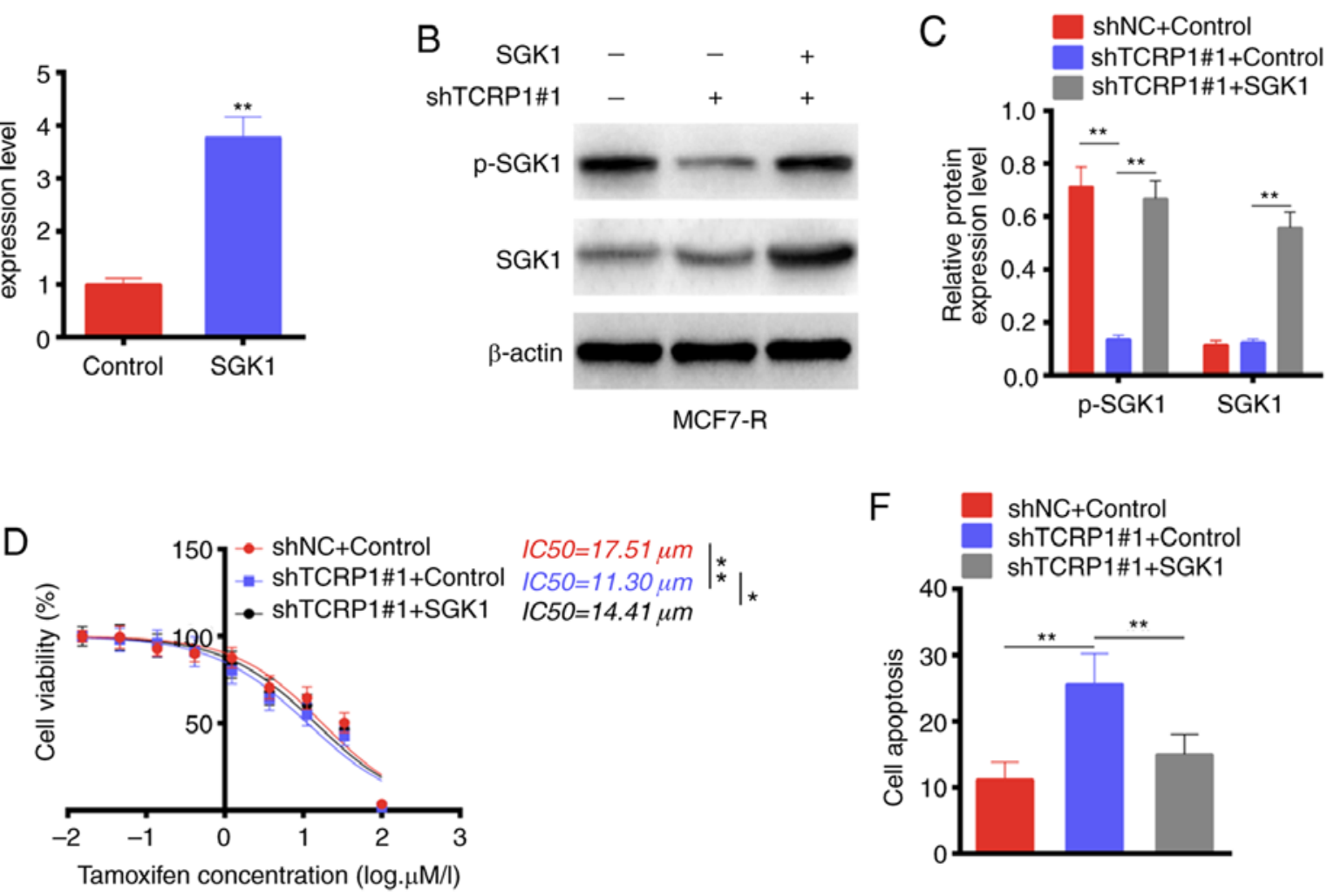
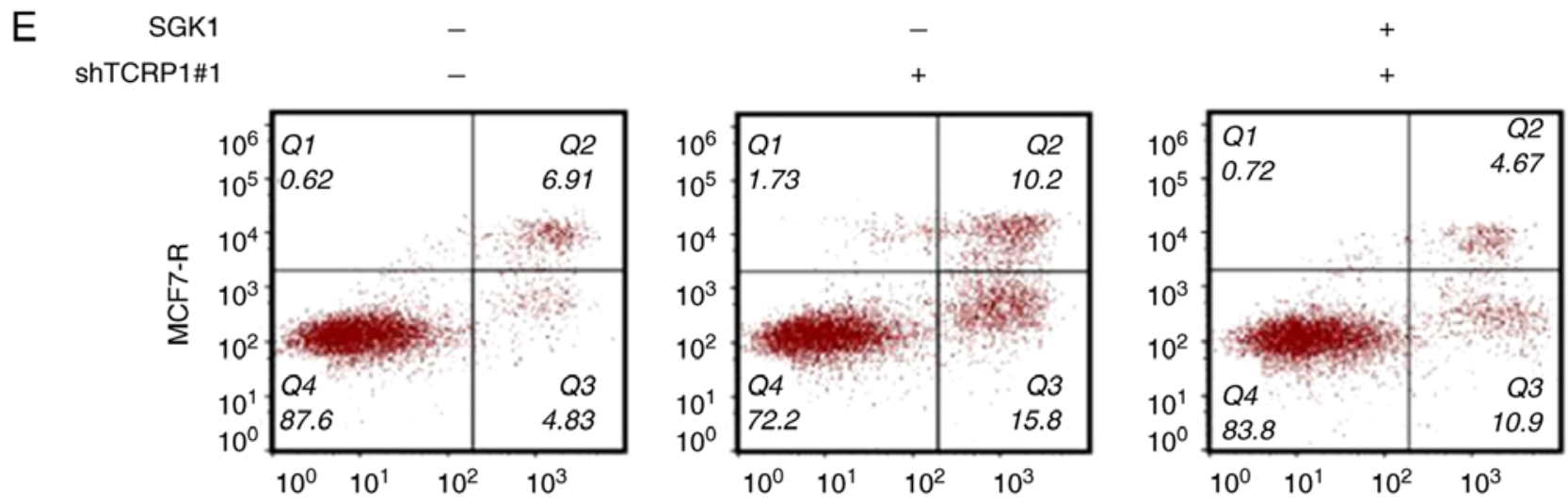

Figure 5. TCRP1 induces tamoxifen resistance by activating SGK1. MCF7-R cells were transfected with a co-transfection system with SGK1 expression vector in combination with the TCRP1 shRNA into MCF7-R cells or transfected with shRNA for TCRP1 or control. (A) The effectiveness of SGK1 overexpression was detected by q-PCR. (B and C) The expression levels of p-SGK1 and SGK1 were detected by western blotting. (D) Cell viability was detected by MTT assay. (E and F) Cell apoptosis was detected by flow cytometry with Annexin V-FITC/PI dual staining. ${ }^{* *} \mathrm{P}<0.01$. TCRP1, tongue cancer resistance-related protein1; SGK1, glucocorticoid-inducible kinase 1.

Moreover, it was observed that TCRP1 induced tamoxifen resistance by phosphorylating PDK1 and further activating PDK1/SGK1 signaling. Therefore, the present study provides strong evidence demonstrating that TCRP1 induces tamoxifen resistance possibly by promoting the activation of SGK1 in MCF7-R cells.

Clinical studies have revealed that high expression of TCRP1 was related to a poor prognosis for patients who experienced OSCC radioresistance (15). In addition, TCRP1 expression was observed to be significantly increased in lung cancer tissues and ovarian cancer tissues, and it could mediate DDP and L-OHP resistance in the cell lines of the two types of cancer (21). Moreover, consistent with upregulated TCRP1, c-Myc was also upregulated in both multidrug-resistant tongue cancer cell line Tca8113/PYM and cisplatin-resistant lung cancer cell line A549/DDP, compared with their parental cells, respectively (22). In the present study, the findings revealed that TCRP1 expression was increased in tamoxifen-resistant breast cancer tissues compared with the primary breast cancer tissues in vivo. Consistently, in vitro TCRP1 expression was also increased in tamoxifen-resistant breast cancer cell line MCF7-R compared with the primary breast cancer cell line MCF-7. These data revealed that TCRP1 may be a pivotal regulator in mediating tamoxifen resistance in MCF-7 cells.

MCF-7 cells are widely used in studying acquired tamoxifen resistance, since it is not only a stable breast cancer cell line, but it is also the most characterized cell line (23-27). 

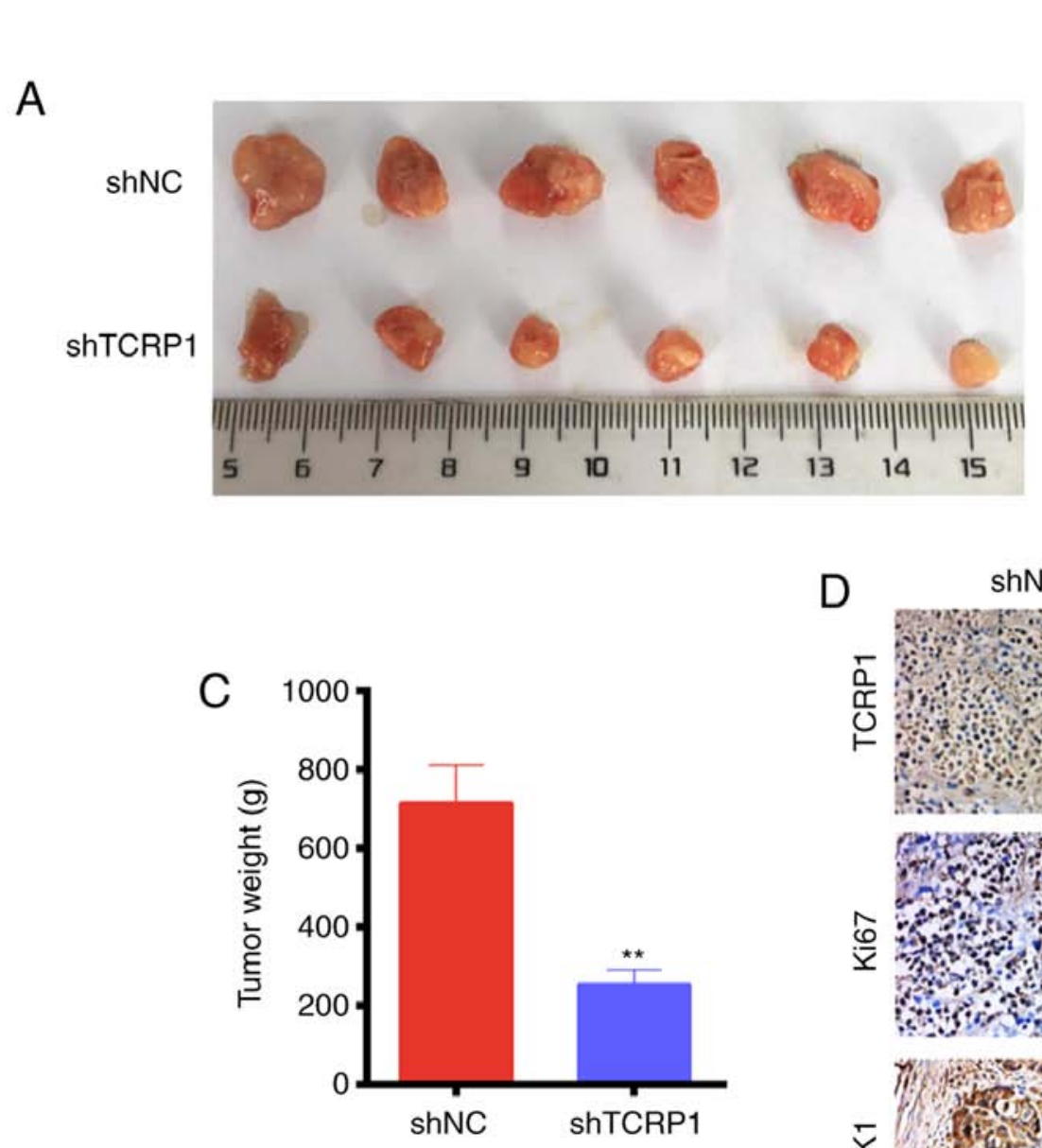

B

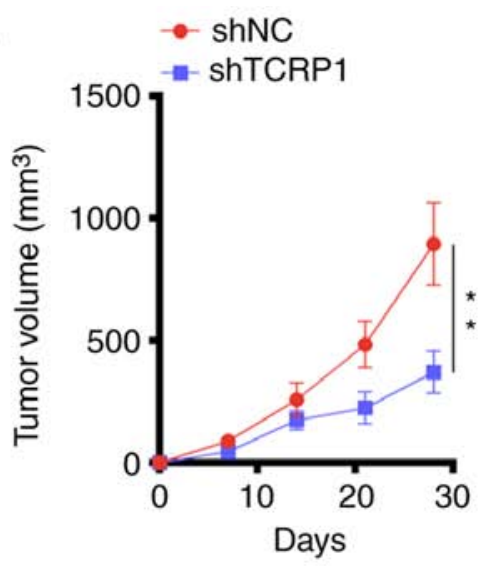

D

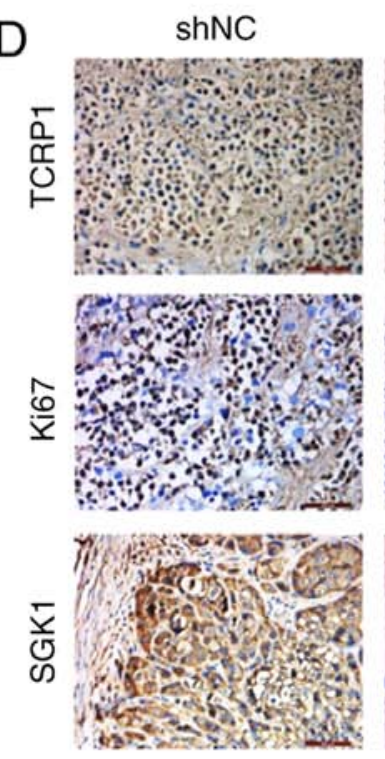

ShTCRP1\#1

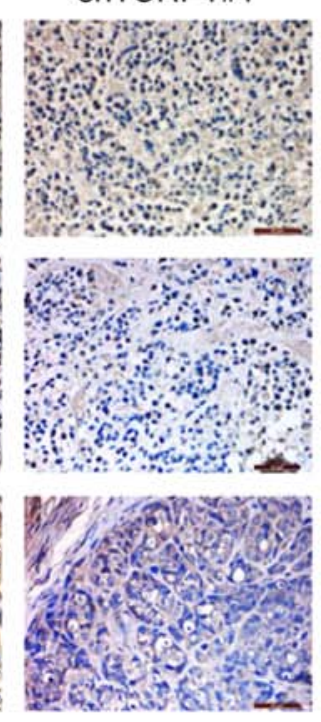

Figure 6. Knockdown of TCRP1 inhibits oncogenesis of MCF7-R cells in nude mice. MCF7-R cells were treated with shRNA for TCRP1 and shRNA control, and then subcutaneously injected into the nude mice. (A and B) Tumor growth was analyzed by measuring the tumor volume. (C) Tumors were excised and weighed. (D) Pathological sections in tumor tissues were subjected to immunohistochemistry with TCRP1, Ki67 and SGK1 antibodies. "* P $<0.01$. TCRP1, tongue cancer resistance-related protein1; SGK1, glucocorticoid-inducible kinase 1.

MCF7-R cells are characterized by activating another survival signal pathway to support breast cancer growth and inhibit the apoptotic-promoting effect of tamoxifen (23). In addition, TCRP1 plays a tumor-promoting role in carcinogenesis and cancer progression. Previous studies reported that overexpressed TCRP1 promoted cell proliferation, cell growth, and cell cycle progression in NIH/3T3 cells. Moreover, upregulated TCRP1 promoted tumorigenesis of NIH/3T3 cells in nude mice (17). It has been reported that TCRP1-proficient cells exhibit an improved survival chance by increasing the levels of antiapoptotic proteins and decreasing the level of proapoptotic proteins (15). However, the function of TCRP1 in tamoxifen-resistant breast cancer is still unknown. Thus, the role of TCRP1 in MCF7-R cells was investigated. The present results revealed that knockdown of TCRP1 decreased the resistance of MCF7-R cells to tamoxifen by inhibiting MCF7-R cell growth and promoting cell apoptosis.

Studies on the mechanisms of tamoxifen resistance demonstrated that tumor cells recruit various signaling pathways to develop drug resistance $(28,29)$. It has been reported that TCRP1 promoted NIH/3T3 cell transformation by over-activating PDK1 and AKT1 (17). In addition, gene amplification or aberrant phosphorylation in the cytosol and nucleus may lead to aberrant PDK1 expression in human cancers such as in colon and breast cancer (30-33). The results revealed that TCRP1 regulated the phosphorylation of PDK1. Specifically, the level of p-PDK1 was significantly increased after TCRP1 overexpression, which was contrary to the knockdown of TCRP1. Although the downstream of phosphorylated PDK1 after TCRP1 stimulation remained unclear, recently, some studies have revealed that PDK1/SGK1 signaling plays multiple roles in a variety of physiological processes such as cell growth, proliferation and survival (20) (34-37). For instance, PDK1 activity was suppressed to inhibit downstream SGK1 phosphorylation and activity of BYL719-resistant cells (20). Hence, the correlations among tamoxifen resistance and PDK1 and SGK1 under TCRP1 stimulation were analyzed in the present study, and the present data indicated that TCRP1 led to tamoxifen resistance by regulating the phosphorylation of PDK1, which further activated SGK1.

It should also be noted that there are four limitations in the present study. Firstly, the correlation between TCRP1 and 
the prognosis of tamoxifen-resistant breast cancer patients was not investigated. Secondly, the effects of TCRP1 on various types of breast cancer cell lines should be studied, to confirm and render the present results more convincing. Thirdly, overexpressing TCRP1 in MCF-7 cells should be performed to observe the effect of TCRP1 on the sensitivity of the breast cancer cells to tamoxifen and the phosphorylation of SGK1. Finally, the clinical data of patients, including ER, PR, HER2 were not obtained in the tissues of patients.

In conclusion, the present data strongly support that TCRP1 contributes to tamoxifen resistance possibly through the activation of the PDK1/SGK1 signaling pathway in MCF7-R cells. The data provide new insights into chemoresistance and provide a novel therapeutic approach that may allow us to prevent tamoxifen resistance in breast cancer in the future.

\section{Acknowledgements}

Not applicable.

\section{Funding}

No funding was received.

\section{Availiability of data and materials}

The analyzed data sets generated during the study are available from the corresponding author on reasonable request.

\section{Authors' contributions}

$\mathrm{SZ}$ and XL performed the experiments and the data analysis. $\mathrm{JL}$ and $\mathrm{XZ}$ wrote the initial draft of the manuscript, conceived and designed the experiments. LY and LH contributed to the literature search, obtained the tumor and tissues with clinical information where it pertained. All authors revised the manuscript. All authors read and approved the final manuscript and agree to be accountable for all aspects of the research in ensuring that the accuracy or integrity of any part of the work are appropriately investigated and resolved.

\section{Ethics approval and consent to participate}

The experimental protocol was approved by the Ethical Committee of Wuzhong People's Hospital, Suzhou, China. The breast cancer tissues were collected from Wuzhong People's Hospital with written informed consent and permission from the Institutional Review Board. All patients provided written informed consent. All animal studies were approved by the Animal Experimentation Ethics Committee of Wuzhong People's Hospital.

\section{Patient consent for publication}

Not applicable.

\section{Competing interests}

The authors declare that they have no competing interests.

\section{References}

1. Ginsburg O, Bray F, Coleman MP, Vanderpuye V, Eniu A, Kotha SR, Sarker M, Huong TT, Allemani C, Dvaladze A, et al: The global burden of women's cancers: A grand challenge in global health. Lancet 389: 847-860, 2017.

2. Bray F, Ferlay J, Soerjomataram I, Siegel RL, Torre LA and Jemal A: Global cancer statistics 2018: GLOBOCAN estimates of incidence and mortality worldwide for 36 cancers in 185 countries. CA Cancer J Clin 68: 394-424, 2018.

3. Hayes EL and Lewis-Wambi JS: Mechanisms of endocrine resistance in breast cancer: An overview of the proposed roles of noncoding RNA. Breast Cancer Res 17: 40, 2015.

4. Shi Y, Zhao Y, Zhang Y, AiErken N, Shao N, Ye R, Lin Y and Wang S: AFF3 upregulation mediates tamoxifen resistance in breast cancers. J Exp Clin Cancer Res 37: 254, 2018.

5. Merikhian P, Ghadirian R, Farahmand L, Mansouri S and Majidzadeh-A K: MUC1 induces tamoxifen resistance in estrogen receptor-positive breast cancer. Expert Rev Anticancer Ther 17: 607-613, 2017.

6. Jiang Q, Zheng S and Wang G: Development of new estrogen receptor-targeting therapeutic agents for tamoxifen-resistant breast cancer. Future Med Chem 5: 1023-1035, 2013.

7. Rao RD and Cobleigh MA: Adjuvant endocrine therapy for breast cancer. Oncology (Williston Park) 26: 541-547, 550, 552, 2012.

8. Jordan VC and O'Malley BW: Selective estrogen-receptor modulators and antihormonal resistance in breast cancer. J Clin Oncol 25: 5815-5824, 2007.

9. Egeland NG, Lunde S, Jonsdottir K, Lende TH, Cronin-Fenton D, Gilje B, Janssen EA and Søiland H: The role of MicroRNAs as predictors of response to tamoxifen treatment in breast cancer patients. Int J Mol Sci 16: 24243-24275, 2015.

10. Nass $\mathrm{N}$ and Kalinski T: Tamoxifen resistance: From cell culture experiments towards novel biomarkers. Pathol Res Pract 211: 189-197, 2015.

11. Nass N, Bromme HJ, Hartig R, Korkmaz S, Sel S, Hirche F, Ward A, Simm A, Wiemann S, Lykkesfeldt AE, et al: Differential response to $\alpha$-oxoaldehydes in tamoxifen resistant MCF-7 breast cancer cells. PLoS One 9: e101473, 2014.

12. Viedma-Rodriguez R, Baiza-Gutman L, Salamanca-Gomez F, Diaz-Zaragoza M, Martínez-Hernández G, Ruiz Esparza-Garrido R, Velázquez-Flores MA and Arenas-Aranda D: Mechanisms associated with resistance to tamoxifen in estrogen receptor-positive breast cancer (review). Oncol Rep 32: 3-15, 2014.

13. Gu Y, Fan S, Xiong Y, Peng B, Zheng G, Yu Y, Ouyang Y and $\mathrm{He} \mathrm{Z}$ : Cloning and functional characterization of TCRP1, a novel gene mediating resistance to cisplatin in an oral squamous cell carcinoma cell line. FEBS Lett 585: 881-887, 2011.

14. Peng B, Gu Y, Xiong Y, Zheng G and He Z: Microarray-assisted pathway analysis identifies MT1X \& NFKB as mediators of TCRP1-associated resistance to cisplatin in oral squamous cell carcinoma. PLoS One 7: e51413, 2012.

15. Gu Y, Fan S, Liu B, Zheng G, Yu Y, Ouyang Y and He Z: TCRP1 promotes radioresistance of oral squamous cell carcinoma cells via Akt signal pathway. Mol Cell Biochem 357: 107-113, 2011.

16. Liu X, Wang C, Gu Y, Zhang Z, Zheng G and He Z: TCRP1 contributes to cisplatin resistance by preventing Pol $\beta$ degradation in lung cancer cells. Mol Cell Biochem 398: 175-183, 2015.

17. Wang C, Liu H, Qiu Q, Zhang Z, Gu Y and He Z: TCRP1 promotes NIH/3T3 cell transformation by over-activating PDK1 and akt1. Oncogenesis 6: e323, 2017.

18. Lin YS, Lin YY, Yang YH, Lin CL, Kuan FC, Lu CN, Chang GH, Tsai MS, Hsu CM, Yeh RA, et al: Antrodia cinnamomea extract inhibits the proliferation of tamoxifen-resistant breast cancer cells through apoptosis and skp2/microRNAs pathway. BMC Complement Altern Med 18: 152, 2018.

19. Du J, Yang M, Chen S, Li D, Chang Z and Dong Z: PDK1 promotes tumor growth and metastasis in a spontaneous breast cancer model. Oncogene 35: 3314-3323, 2016.

20. Castel P, Ellis H, Bago R, Toska E, Razavi P, Carmona FJ, Kannan S, Verma CS, Dickler M, Chandarlapaty S, et al: PDK1-SGK1 Signaling sustains AKT-independent mTORC1 activation and confers resistance to PI3K $\alpha$ inhibition. Cancer Cell 30: 229-242, 2016.

21. Liu X, Feng M, Zheng G, Gu Y, Wang C and He Z: TCRP1 expression is associated with platinum sensitivity in human lung and ovarian cancer cells. Oncol Lett 13: 1398-1405, 2017. 
22. Jia X, Zhang Z, Luo K, Zheng G, Lu M, Song Y, Liu H, Qiu H and He Z: TCRP1 transcriptionally regulated by c-Myc confers cancer chemoresistance in tongue and lung cancer. Sci Rep 7: $3744,2017$.

23. Zhou C, Zhong Q, Rhodes LV, Townley I, Bratton MR, Zhang Q Martin EC, Elliott S, Collins-Burow BM, Burow ME and Wang G: Proteomic analysis of acquired tamoxifen resistance in MCF-7 cells reveals expression signatures associated with enhanced migration. Breast Cancer Res 14: R45, 2012.

24. Fan P, Yue W, Wang JP, Aiyar S, Li Y, Kim TH and Santen RJ: Mechanisms of resistance to structurally diverse antiestrogens differ under premenopausal and postmenopausal conditions: Evidence from in vitro breast cancer cell models. Endocrinology 150: 2036-2045, 2009.

25. Knowlden JM, Hutcheson IR, Jones HE, Madden T, Gee JM, Harper ME, Barrow D, Wakeling AE and Nicholson RI: Elevated levels of epidermal growth factor receptor/c-erbB2 heterodimers mediate an autocrine growth regulatory pathway in tamoxifen-resistant MCF-7 cells. Endocrinology 144 1032-1044, 2003.

26. Leung EY,Kim JE, Askarian-Amiri M, Joseph WR, McKeage MJ and Baguley BC: Hormone resistance in two MCF-7 breast cancer cell lines is associated with reduced mTOR signaling, decreased glycolysis, and increased sensitivity to cytotoxic drugs. Front Oncol 4: 221, 2014.

27. Nawata H, Bronzert D and Lippman ME: Isolation and characterization of a tamoxifen-resistant cell line derived from MCF-7 human breast cancer cells. J Biol Chem 256: 5016-5021, 1981.

28. Musgrove EA and Sutherland RL: Biological determinants of endocrine resistance in breast cancer. Nat Rev Cancer 9: 631-643, 2009.
29. Gee JM, Shaw VE, Hiscox SE, McClelland RA, Rushmere NK and Nicholson RI: Deciphering antihormone-induced compensatory mechanisms in breast cancer and their therapeutic implications. Endocr Relat Cancer 13 (Suppl 1) S77-S88, 2006.

30. Raimondi $C$ and Falasca M: Targeting PDK1 in cancer. Curr Med Chem 18: 2763-2769, 2011.

31. Peng B, Yi S, Gu Y, Zheng G and He Z: Purification and biochemical characterization of a novel protein-tongue cancer chemotherapy resistance-associated protein1 (TCRP1). Protein Expr Purif 82: 360-367, 2012.

32. Parsons R: Human cancer, PTEN and the PI-3 kinase pathway. Semin Cell Dev Biol 15: 171-176, 2004.

33. Lin HJ, Hsieh FC, Song H and Lin J: Elevated phosphorylation and activation of PDK-1/AKT pathway in human breast cancer. Br J Cancer 93: 1372-1381, 2005.

34. Di Blasio L, Gagliardi PA, Puliafito A and Primo L: Serine/threonine kinase 3-phosphoinositide-dependent protein kinase-1 (PDK1) as a key regulator of cell migration and cancer dissemination. Cancers (Basel) 9: E25, 2017.

35. Bayascas JR: PDK1: The major transducer of PI 3-kinase actions Curr Top Microbiol Immunol 346: 9-29, 2010.

36. Fyffe $\mathrm{C}$ and Falasca M: 3-Phosphoinositide-dependent protein kinase-1 as an emerging target in the management of breast cancer. Cancer Manag Res 5: 271-280, 2013.

37. Emmanouilidi A and Falasca M: Targeting PDK1 for chemosensitization of cancer cells. Cancers (Basel) 9: E140, 2017.

This work is licensed under a Creative Commons Attribution-NonCommercial-NoDerivatives 4.0 International (CC BY-NC-ND 4.0) License. 